\title{
UNITARY GROUPS AND DIFFERENTIAL OPERATORS
}

\author{
ROBERT M. KAUFFMAN
}

\begin{abstract}
Unitary groups generated by differential operators have special properties that can be used to study completeness of the set of eigenvectors of the infinitesimal generator.

Unitary groups also occur in differential operator theory in another manner, associated with unitary equivalence of differential operators. We discuss what happens to $A_{t}=U_{t}^{-1} A U_{t}$ as $t$ approaches infinity provided that $A_{t}$ is a differential operator for all $t$ and $A$ has certain properties.
\end{abstract}

By a differential expression $T$ we mean a formal expression of type $\sum_{0}^{n} a_{i} D^{i}$, where $D$ denotes $i d / d x$ and the $a_{i}$ are $C^{\infty}$ complex valued functions with $a_{n}$ a nonzero constant function. The domain of the $a_{i}$ will be $(-\infty, \infty)=R$.

Associated with an ordinary differential expression we have many possible operators in the space $L_{2}(-\infty, \infty)$. We shall consider only operators which contain the minimal operator $T_{0}$ and are contained in the maximal operation $T_{M}$. The definition of these operators can be found in Goldberg [1]. By a differential operator, then, we mean an operator in $L_{2}(-\infty, \infty)$ which is in between $T_{0}$ and $T_{M}$ for some differential expression $T$.

Our first lemma is proved by using an argument used in Lax and Phillips [3]. Recall that selfadjoint operators generate unitary groups according to Stone's theorem. Here we will deal with selfadjoint operators which are also differential operators. These, of course, can only arise from differential expressions which are equal to their own formal adjoint.

Lемма 1. Let $H$ be a selfadjoint differential operator. Let $U_{t}$ be the unitary group which has infinitesimal generator $i H$, and let $f$ be perpendicular to the eigenvectors of $H$. Then there is a sequence $t_{n}$ approaching infinity such that for any compact subinterval $J$ of $R, \int_{J}\left|U_{t_{n}}\right|^{2}(x) d x$ approaches zero. $t_{n}$ is independent of $f$ and $J$.

Proof. Let $K$ denote the orthogonal complement of the eigenvectors of $H$. Restricting $H$ to $K$, we get a selfadjoint operator $H_{1}$ from a dense subspace of $K$ into $K$. This fact may be seen by making a series of observations, each of which is easy to prove. First, if $P(\Delta)$ is the spectral projection of $H$ corresponding to the Borel set

Received by the editors December 11, 1970.

AMS 1970 subject classifications. Primary 47E05, 34B25.

Key words and phrases. Unitary group, differential operator, eigenvector. 
$\Delta$, and if $P_{K}$ is the projection on $K$, then $P_{K}$ commutes with $P(\Delta)$. Second, if $Q_{n}=P_{n}-P_{-n}$, where $P_{\lambda}$ is the spectral family of projections for $H$, then $\left(H Q_{n}\right) P_{K}=P_{K}\left(H Q_{n}\right)$, which is proved using the first observation and the spectral theorem. Third, if $f$ is in the domain of $H$, then $P_{K} f$ is in the domain of $H$. This can be seen by noting that $Q_{n} g$ approaches $g$ as $n$ approaches infinity, for all $g$ in the Hilbert space. Now if $g$ is in the domain of $H, Q_{n} P_{K} g$ approaches $P_{K} g$ as $n$ approaches infinity, and also $H\left(Q_{n} P_{K} g\right)=\left(H Q_{n}\right) P_{K} g=P_{K}\left(H Q_{n}\right) g$ $=P_{K} Q_{n} H g$ which approaches $P_{K} H g$. Therefore, since $H$ is closed, $P_{K} g$ is in the domain of $H$ and $H P_{K} g=P_{K} H g$. From these three observations, it is not hard to see that $H_{1}$ is a selfadjoint operator in $K$. $H_{1}$ has, of course, no point spectrum.

Now, restricting $U_{t}$ to $K$, we get a unitary group $V_{t}$ on $K$ which has $i H_{1}$ as its infinitesimal generator. But then (see Lax and Phillips $\left[3\right.$, p. 145]), it follows that there is a sequence $t_{n}$ approaching infinity with $V_{t_{n}}$ converging weakly to zero.

Let $T_{J}$ denote the differential expression on $J$ formed by restricting $T$ to $J$, where $T$ is the differential expression associated with $H$. Thus $\left(T_{J}\right)_{M}$ denotes the maximal operator in $L_{2}(J)$ corresponding to $T_{J}$.

Recalling that $J$ is compact, we see that the set of all $f$ in $L_{2}(J)$, such that $\|f\| \leqq 1$ and $\left\|\left(T_{J}\right)_{M} f\right\| \leqq N$, has compact closure in $L_{2}(J)$, for each fixed $N$. This known fact can be proved in several different ways, one of which involves almost a word for word repetition of part of the proof of Theorem 2 .

Now we finish the proof. It suffices to prove the theorem for $f$ in domain $H_{1}$, since domain $H_{1}$ is dense in $K$. But for such $f$, we see that $U_{t_{n}} f$ is also in domain $H_{1}$, where $t_{n}$ is selected so that $V_{t_{n}}$ approaches zero weakly on $K$. Letting $C_{J}$ be the characteristic function of $J$, $C_{J} U_{t_{n}} f$ is in the domain of $\left(T_{J}\right)_{M}$, and $\left(T_{J}\right)_{M} C_{J} f_{n}=C_{J} T f_{n}$. We are identifying $L_{2}(J)$ with a subspace of $L_{2}(R)$ here, and letting $f_{n}$ be $U_{t_{n}} f$. However, since $\|T f\|=\|H f\|=\left\|U_{t_{n}}^{-1} H U_{t_{n}} f\right\|=\left\|H f_{n}\right\|$, it follows that both $C_{J} T f_{n}$ and $C_{J} f_{n}$ are bounded in norm. Thus there is a subsequence $h_{j}$ of $f_{n}$ such that $C_{J} h_{j}$ is convergent in norm to a point of $L_{2}(J)$. But since $f_{n}$ converges weakly to zero, it follows that $C_{J} h_{j}$ converges to zero in norm in $L_{2}(J)$. This is the conclusion of the theorem, because it implies that $C_{J} f_{n}$ approaches zero, since the sequence is contained in a compact set and zero is its only possible cluster point.

TheOREM 1. Let $T=\sum_{1}^{n} T_{i}+V(x)$, where $T$ is a classically selfadjoint differential expression, $V(x)$ is a complex valued function on the reals such that $|V(x)|$ approaches infinity with $|x|$, and $T_{i}$ is a differ- 
ential expression (not necessarily selfadjoint) for each $i$. Let $H$ be $a$ selfadjoint differential operator associated with the differential expression $T$, and suppose that $f$ in domain $H$ implies that $T_{i} f$ is in $L_{2}$ for each $i$. Then the eigenvectors of $H$ form an orthonormal basis for $L_{2}(R)$.

Proof. Suppose $f$ is in the domain of $H$, and $f$ is perpendicular to the linear span of the eigenvectors of $H$. We know from part of the proof of Lemma 1, that if the linear span of the eigenvectors is not dense, there is such an $f$ with $\|f\|=1$.

Select $t_{n}$ as in Lemma 1. Let $f_{n}=U_{t_{n}} f$. Then $\left\|V f_{n}\right\|$ approaches infinity, by Lemma 1. But also $\left\|f_{n}\right\|=\|f\|$, and $\left\|H f_{n}\right\|=\|H f\|$. If we can show the existence of a number $N$ such that, for all $T_{i},\left\|T_{i} f_{n}\right\|$ $\leqq N\left(\left\|f_{n}\right\|+\left\|H f_{n}\right\|\right)$, we have a contradiction. We shall show it for each $T_{i}$ separately.

Consider the domain of $H$, with norm $\|g\|_{1}=\|g\|+\|H g\|$. This norm makes domain $H$ into a Banach space. If we fix $i$, and map $g$ to $T_{i} g$, where $g$ is given \|\|$_{1}$ and $T_{i} g$ is given $L_{2}$ norm, the resulting linear transformation $W_{i}$ has a closed graph. To see this, suppose that $\left\|g_{n}-g\right\|_{1}$ approaches zero, and $T_{i} g_{n}$ is a Cauchy sequence in $L_{2}$ norm, we must show that $T_{i} g_{n}$ approaches $T_{i} g$. However, this follows from the fact that $\left(T_{i}\right)_{M}$ is a closed operator. By the closed graph theorem, then, $W_{i}$ is bounded for all $i$. Thus $N$ may be selected. This completes the proof.

Now we consider the question of unitary equivalence. If $K$ is an operator, and $U_{t}$ is a unitary group which carries the domain of $K$ onto itself, then $U_{t}^{-1} K U_{t}=K_{t}$ forms a one parameter family of operators. One might look for some sort of asymptotic behavior of $K_{t}$. For example, if we consider $K=T_{0}$, where $T=-d^{2} / d x^{2}+V(x)$, and $V(x)$ has compact support, and if $U_{t}$ is the unitary group of right translation operators, then on any closed interval $K_{t}$ is the operator $-d^{2} / d x^{2}$ for $t$ large enough. The next theorem negates the possibility of stable asymptotic behavior of this type for certain $T$, provided that the unitary group is generated by an operator with no point spectrum.

We introduce some notation beforehand. If $T$ is a differential expression, we let $T_{l}$ denote the minimal operator on the left half line and $T_{r}$ denote the minimal operator on the right half line induced by $T$. Let $T_{1}$ denote the restriction of $T_{M}$ to $D\left(T_{l}\right)+D\left(T_{r}\right)$, where $D(A)$ denotes the domain of the operator $A . T_{0}$ is a finite dimensional extension of $T_{1}$.

Also, if $T$ is a differential expression, we write $\|T\|_{I}$ $=\max _{0 \leqq i \leqq n} \sup _{x \in 1}\left|a_{i}(x)\right|$ for any closed and bounded interval $I$. 
THEOREM 2. Let $A$ be a differential operator generated by the differential expression $T$. Suppose that $U_{t}$ is a one parameter unitary group which takes $D(A)$ into $D(A)$ for all $t$. If iH is the infinitesimal generator of $U_{t}$, we suppose $H$ has no point spectrum. We suppose that $U_{t}^{-1} A U_{t}$ is a differential operator $A_{t}$ generated by a differential expression $T_{t}$ for each $t$. Finally, we suppose that $T_{r}^{-1}$ and $T_{l}^{-1}$ are completely continuous operators from their ranges into $L_{2}$. Then there is a sequence $t_{n}$ approaching infinity such that $\left\|T_{t_{n}}\right\|_{I}$ approaches infinity for all compact subintervals $I$ of the reals.

REMARK. The property that $T_{r}^{-1}$ and $T_{l}^{-1}$ be completely continuous has been investigated by Kauffman [2].

Proof. We first prove a preliminary fact. We show that, for a fixed $N$, the set of all $f$ such that $\|A f\| \leqq N$ and $\|f\| \leqq 1$ has compact closure in $L_{2}$. The proof follows. Call the set in question $S_{N}$.

Let $K$ be the null space of $A$. Decompose $D(A)$ in to $D\left(T_{1}\right)+S+K$, where $S$ is finite dimensional and the three spaces are linearly independent. Any $f$ in $D(A)$ can be written as $f_{1}+f_{2}+f_{3}$, where $f_{1}$ is in $D\left(T_{1}\right), f_{2}$ is in $S$, and $f_{3}$ is in $K$. Then range $A$ is range $T_{1}+A(S)$, and these two spaces are linearly independent.

Let $P(g+h)=g$, where $g+h$ is in range $A, g$ is in range $T_{1}$, and $h$ is in $A(S)$. Since $S$ is finite dimensional and range $T_{1}$ is closed, $P$ is a bounded linear transformation of range $A$ into range $T_{1}$. Let $\|P\|=M_{1}$, and let $\left\|T_{1}^{-1}\right\|=M_{2}$.

We see that if $f$ is in $D(A)$, then $\left\|f_{1}\right\| \leqq M_{1} M_{2}\|A f\|$. Therefore, if $f$ is in $S_{N},\left\|f_{1}\right\| \leqq M_{1} M_{2} N$. From this it follows that $\left\|f_{2}+f_{3}\right\| \leqq M_{1} M_{2} N$ +1 . However, $T_{1}^{-1}$ is completely continuous, and the set of all $f_{1}$, where $f$ ranges over $S_{N}$, is contained in $T_{1}^{-1}\left(B_{M_{2} N}\right)$, where $B_{r}$ is the ball of radius $r$ in range $T_{1}$. Thus the set of $f_{1}$ has compact closure. Also the set of $f_{2}+f_{3}$ is contained in a ball of the finite dimensional space $S+K$, and thus the set of $f_{2}+f_{3}$ has compact closure as well. This proves our claim that $S_{N}$ has compact closure.

Now select $t_{n}$ such that $U_{t_{n}}$ approaches zero weakly. Then, if for some $I$ there were a subsequence $t_{n(j)}$ such that $\left\|T_{t_{n(j)}}\right\|_{I}$ remains bounded, pick $f$ such that $\|f\|=1$ and $f$ is in $C_{0}^{\infty}(I)$. Then $\left\|U_{t_{n(j)}}^{-1} A U_{t_{n(j)}} f\right\|=\left\|A U_{t_{n(j)}} f\right\|$. But by definition, $U_{t}^{-1} A U_{t}=A_{t}$, so $\left\|A U_{t_{n(j)}} f\right\|=\left\|A_{t_{n(j)}} f\right\|$. By assumption $\left\|A_{t_{n(j)}} f\right\|$ remains bounded, so the same is true for $\left\|A U_{t_{n(j)}} f\right\|$. But $\left\|U_{t_{n(j)}} f\right\|=1$, so there is a subsequence of the sequence $U_{t_{n(j)}} f$ which converges to $g$ in norm, by the fact that all these are contained in some $S_{N}$ if $N$ is large enough. But since $U_{t_{n}}$ approaches zero in the weak operator topology, $g$ must be zero. This is impossible, and the proof is finished. 


\section{REFERENCES}

1. S. Goldberg, Unbounded linear operators: Theory and applications, McGrawHill, New York, 1966. MR 34 \#580.

2. R. M. Kauffman, Operator theory of a class of ordinary differential expressions, J. Differential Equations (submitted).

3. P. Lax and R. S. Phillips, Scattering theory, Pure and Appl. Math., vol. 26, Academic Press, New York, 1967. MR 36 \#530.

Western Washington State College, Bellingham, Washington 98225 